\title{
Optimal dividend and reinsurance in the presence of two reinsurers
}

\author{
Mi Chen and Kam Chuen Yuen
}

Abstract: In this paper, the optimal dividend (subject to transaction costs) and reinsurance (with two reinsurers) problem is studied in the limit diffusion setting. It is assumed that transaction costs and taxes are required when dividends occur, and that the premiums charged by two reinsurers are calculated according to the exponential premium principle with different parameters, which makes the stochastic control problem nonlinear. The objective of the insurer is to determine the optimal reinsurance and dividend policy so as to maximize the expected discounted dividends until ruin. The problem is formulated as a mixed classical-impulse stochastic control problem. Explicit expressions for the value function and the corresponding optimal strategy are obtained. Finally, a numerical example is presented to illustrate the impact of the parameters associated with the two reinsurers' premium principle on the optimal reinsurance strategy.

Keywords: dividend; reinsurance; transaction costs; exponential premium principle; optimal reinsurance with two reinsurers

\section{Introduction}

In the actuarial literature, insurance risk model with dividend payments was first considered by de Finetti [7]. In his paper, the optimal expected discounted sum of dividend payments until the time of ruin was studied in a simple discrete time model. Since then, many researchers carried out similar analysis for various risk models with more general and realistic features. For example, optimal dividend problems with transaction costs and controlled risk exposure can be found in Cadenillas et al. [3], He and Liang [11, 12], Løkka and Zervos [15], Bai et al. [2], Meng and Siu [16, 17], Scheer and Schmidli [21], Peng et al. [20] and Guan and Liang [9].

In most of the literature, premium is assumed to be calculated via the expected value principle for mathematical convenience. However, it is natural to argue that two risks with same mean may look very different from each other, and hence the associated premiums should also be different. The exponential premium principle, which is the so-called zero utility principle, plays an important role in insurance mathematics and 
actuarial practice. It has many nice properties, including additivity with respect to independent risks. It is also widely used in mathematical finance to price various insurance products in the market. We refer the readers to Young and Zariphopoulou [26], Young [25], Moore and Young [18] and Musiela and Zariphopoulou [19]. For the optimal reinsurance problems under other premium principles, one can see Schmidli [22], Young [24], Kaluszka [13, 14], Zhou and Yuen [27] and Yao et al. [23].

In practice, insurance companies often purchase reinsurance to reduce the risk of their insurance portfolios. For simplicity, it is usually assumed in the literature that an insurer can only buy reinsurance from one reinsurer. However, it is commonly seen that some insurance company would like to diversify its risk by purchasing reinsurance from multiple reinsurance companies who may have different risk attitudes. Thus, it is meaningful to study the optimal reinsurance models with multiple reinsurers. Recently, optimal reinsurance problems with multiple reinsurers under the criterion of minimizing value at risk ( $\mathrm{VaR})$ or conditional value at risk $(\mathrm{CVaR})$ of the insurer's total risk exposure were studied by Asimit et al. [1] and Chi and Meng [5].

Under the exponential premium principle, the optimal dividend problem without transaction costs is investigated in Chen et al. [4], where only one reinsurer is considered. In this paper, we study the optimal dividend problem subject to transaction costs and optimal reinsurance with two reinsurers in the framework of diffusion model. We assume that the premiums charged by the two reinsurers are calculated according to the exponential premium principle with different parameters, which is closely related to a kind of nonlinear classical-impulse stochastic control problem. Under the exponential premium principle, the risk control becomes nonlinear which makes the problem more complicated than that under the expected value premium principle. In view of the complexity, we consider proportional reinsurance only in our study. Our objective is to maximize the expected discounted dividends until ruin. Explicit expressions for the value function and the corresponding optimal strategies are derived.

The rest of this paper is organized as follows. In Section 2, we present the mathematical formulation of the model with proportional reinsurance and dividend payments under the exponential premium principle. In Section 3, we give the quasi-variational inequalities (QVI) and the verification theorem of the problem. In Section 4, we give the solution to the optimization problem. We then give some comments in Section 5 , and provide a numerical example in Section 6. 


\section{The Model}

In this paper, all stochastic quantities are defined on a large enough complete probability space $\left(\Omega, \mathcal{F}, \mathcal{F}_{t}, \mathbf{P}\right)$, where the filtration $\mathcal{F}_{t}$ represents the information available at time $t$ and any decision made is based on this information.

Our results will be formulated within the controlled diffusion model. But we start with the classical Cramér-Lundberg model, in which the surplus process of an insurer is given by $U_{t}=x+c t-\sum_{i=1}^{N_{t}} Y_{i}$, where $x \geq 0$ is the initial surplus, $c>0$ is the premium rate, $\{N(t), t \geq 0\}$ is a homogeneous Poisson process with intensity $\lambda$, and $\left\{Y_{i}, i \geq\right.$ $1\}$ is a sequence of positive i.i.d. random variables with common distribution $F(y)$. We denote by $\mu_{1}=E\left(Y_{i}\right)$ its mean and by $M_{Y}(r)=E\left(e^{r Y_{i}}\right)$ its moment generating function. It is usually assumed that the Cramér-Lundberg conditions hold, i.e., there exists $0<r_{\infty} \leq \infty$ such that $M_{Y}(r)<\infty$ if $r<r_{\infty}$ and that $\lim _{r \rightarrow r_{\infty}} M_{Y}(r)=+\infty$.

Here, we assume that the insurer is allowed to reduce the risk by purchasing proportional reinsurance with two reinsurers. Specifically, for a claim $Y$ occurring at time $t$, the first reinsurer pays $\left(1-b_{t}\right) Y$, the second reinsurer pays $\left(1-u_{t}\right) b_{t} Y$, and the insurer itself pays $u_{t} b_{t} Y$. We denote by $C\left(b_{t}, u_{t}\right)$ the net income rate of the insurer at time $t$. Then the surplus process in the presence of proportional reinsurance (for fixed $b$ and $u$ ) can be written as

$$
U_{t}^{b, u}=x+C(b, u) t-\sum_{i=1}^{N_{t}} u b Y_{i} .
$$

It is well known that (2.1) can be approximated by a pure diffusion model $X_{t}^{b, u}$ with the same drift and volatility. Specifically, if $b$ and $u$ change with time and are stochastic, then the controlled surplus process $X_{t}^{b, u}$ with the strategy $\left(b_{t}, u_{t}\right)$ satisfies

$$
d X_{t}^{b, u}=\left[C\left(b_{t}, u_{t}\right)-\lambda u_{t} b_{t} \mu_{1}\right] d t+\sqrt{\lambda \mu_{2}} u_{t} b_{t} d W_{t}
$$

with $X_{0}^{b, u}=x$, where $\left\{W_{t}, t \geq 0\right\}$ is a standard Brownian motion, and $\mu_{1}, \mu_{2}$ are the first two moments of $Y_{i}$.

In addition to purchasing proportional reinsurance, the insurance portfolio pays dividends to its shareholders under some dividend strategy. Here, we take into account a fixed transaction cost $K>0$ and a tax rate $1-k(0<k<1)$ which are incurred each time the dividend is paid out. Since every dividend results in a fixed transaction cost $K>0$, the insurance company should not pay out dividends continuously. Instead, it should pay dividends at some discrete time points. Then, a strategy is described by

$$
\alpha=\left(b_{t} ; u_{t} ; \tau_{1}, \tau_{2}, \ldots, \tau_{n}, \ldots ; \xi_{1}, \xi_{2}, \ldots, \xi_{n}, \ldots\right)
$$


where $\tau_{n}$ and $\xi_{n}$ denote the times and amounts of dividends. For a strategy $\alpha$, we denote by $X_{t}^{\alpha}$ the associated surplus process whose dynamics is given by

$$
X_{t}^{\alpha}=x+\int_{0}^{t} \mu\left(b_{s}, u_{s}\right) d s+\int_{0}^{t} \sqrt{\lambda \mu_{2}} b_{s} u_{s} d W_{s}-\sum_{n=1}^{\infty} I_{\left(\tau_{n}<t\right)} \xi_{n},
$$

where

$$
\mu\left(b_{s}, u_{s}\right)=C\left(b_{s}, u_{s}\right)-\lambda u_{s} b_{s} \mu_{1}
$$

The ruin time of the controlled process $X_{t}^{\alpha}$ is than defined as

$$
\tau^{\alpha}=\inf \left\{t \geq 0: X_{t}^{\alpha}<0\right\}
$$

Definition 2.1. A strategy $\alpha$ is said to be admissible if

(i) $b_{t}$ and $u_{t}$ are $\left\{\mathcal{F}_{t}\right\}_{t \geq 0}$-adapted processes with $0 \leq b_{t} \leq 1,0 \leq u_{t} \leq 1$ for all $t \geq 0$.

(ii) $\tau_{n}$ is a stopping time with respect to $\left\{\mathcal{F}_{t}\right\}_{t \geq 0}$ and $0 \leq \tau_{1}<\tau_{2}<\cdots<\tau_{n}<\cdots$ a.s.

(iii) $\xi_{n}$ is measurable with respect to $\mathcal{F}_{\tau_{n}-}$ and $0<\xi_{n} \leq X_{\tau_{n}-}^{\alpha}, n=1,2, \ldots$

(iv) $P\left(\lim _{n \rightarrow \infty} \tau_{n} \leq T\right)=0$, for all $T \geq 0$.

The set of all admissible control strategies is denoted by П. For a given admissible strategy $\alpha$, we define the return function as

$V_{\alpha}(x)=E\left[\sum_{n=1}^{\infty} e^{-\delta \tau_{n}}\left(k \xi_{n}-K\right) I_{\left\{\tau_{n}<\tau^{\alpha}\right\}} \mid X_{0-}=x\right]=E_{x}\left[\sum_{n=1}^{\infty} e^{-\delta \tau_{n}}\left(k \xi_{n}-K\right) I_{\left\{\tau_{n}<\tau^{\alpha}\right\}}\right]$,

which represents the expected total discounted dividends received by the shareholders until the ruin time when the initial surplus is $x$, where $\delta>0$ is a priori given discount factor. The objective is to find the optimal return function (or value function), which is defined as

$$
V(x)=\sup _{\alpha \in \Pi} V_{\alpha}(x)
$$

and to find the optimal strategy $\alpha^{*}$ such that $V(x)=V_{\alpha^{*}}(x)$ for all $x \geq 0$.

\section{QVI and verification theorem}

For a function $\phi:[0, \infty) \mapsto[0, \infty)$, we define the maximum operator $\mathcal{M}$ as

$$
\mathcal{M} \phi(x):=\sup \{\phi(x-\eta)+k \eta-K: 0<\eta \leq x\},
$$

and the operator $\mathcal{L}^{b, u}$ as

$$
\mathcal{L}^{b, u} \phi(x):=\frac{1}{2} \lambda \mu_{2} b^{2} u^{2} \phi^{\prime \prime}(x)+\mu(b, u) \phi^{\prime}(x) .
$$


Remark 3.1. For the value function $V(x)$, it is easy to see that $\mathcal{M} V(x) \leq V(x)$.

If the value function of (2.5) is sufficiently smooth, then by standard arguments in stochastic control (see, e.g., Fleming and Soner [8]), the corresponding QVI is given by

$$
\max \left\{\max _{0 \leq b \leq 1,0 \leq u \leq 1} \mathcal{L}^{b, u} V(x)-\delta V(x), \mathcal{M} V(x)-V(x)\right\}=0, \quad x>0,
$$

with boundary condition $V(0)=0$. Given a solution $v(x)$ to (3.1), we can construct the following Markov control strategy.

Definition 3.1. The strategy $\alpha^{v}=\left(b^{v} ; u^{v} ; \tau_{1}^{v}, \tau_{2}^{v}, \cdots, \tau_{n}^{v}, \cdots ; \xi_{1}^{v}, \xi_{2}^{v}, \cdots, \xi_{n}^{v}, \cdots\right)$ is called the QVI strategy associated with $v$ if the associated process $X^{v}$ given by (2.3) with $x \geq 0$ satisfies

$$
\begin{aligned}
& \left(b_{t}^{v}, u_{t}^{v}\right)=\arg \max _{0 \leq b \leq 1,0 \leq u \leq 1} \mathcal{L}^{b, u} v\left(X_{t}^{v}\right) \text { on }\left\{v\left(X_{t}^{v}\right)>\mathcal{M} v\left(X_{t}^{v}\right)\right\}, \\
& \tau_{1}^{v}=\inf \left\{t \geq 0: v\left(X_{t}^{v}\right)=\mathcal{M} v\left(X_{t}^{v}\right)\right\}, \\
& \xi_{1}^{v}=\arg \sup _{0<\eta \leq X_{\tau_{1}^{v}}^{v}}\left\{v\left(X_{\tau_{1}^{v}}^{v}-\eta\right)+k \eta-K\right\}
\end{aligned}
$$

and for every $n \geq 2$,

$$
\begin{aligned}
& \tau_{n}^{v}=\inf \left\{t>\tau_{n-1}^{v}: v\left(X_{t}^{v}\right)=\mathcal{M} v\left(X_{t}^{v}\right)\right\}, \\
& \xi_{n}^{v}=\arg \sup _{0<\eta \leq X_{\tau_{n}^{v}}^{v}}\left\{v\left(X_{\tau_{n}^{v}}^{v}-\eta\right)+k \eta-K\right\} .
\end{aligned}
$$

Throughout this paper, we assume that the reinsurance premium is calculated according to the exponential premium principle. That is, for a risk $U$, the amount of premium $\pi_{a}(U)$ is determined by

$$
\pi_{a}(U)=\frac{1}{a} \ln E\left(e^{a U}\right),
$$

where the constant $a>0$ measures the risk aversion of the reinsurance company. We allow the two reinsurers have different risk aversion, and the parameters for them are $a_{1}$ and $a_{2}$, respectively. Then $\mu\left(b_{t}, u_{t}\right)$ defined in (2.4) becomes

$$
\mu\left(b_{t}, u_{t}\right)=c-\frac{\lambda}{a_{1}}\left(M_{Y}\left(a_{1}\left(1-b_{t}\right)\right)-1\right)-\frac{\lambda}{a_{2}}\left(M_{Y}\left(a_{2}\left(1-u_{t}\right) b_{t}\right)-1\right)-\lambda u_{t} b_{t} \mu_{1} .
$$

Remark 3.2. $\quad$ For the expected premium principle, diversifying between different reinsurers is never optimal for the insurer. The reinsurer with the smallest safety loading will always be the one providing the cheapest insurance, and the insurer will 
always buy reinsurance from this reinsurer. However, for the exponential premium principle, the situation is completely different. For example, for a risk $X$, it is easy to see that $\pi_{a}(X) \geq 2 \pi_{a}\left(\frac{X}{2}\right)$, which means that diversifying the risk between two reinsurers with the same parameter a is always better than sticking with one of them only. Besides, for two reinsurers with parameters $a_{1}$ and $a_{2}\left(a_{1}<a_{2}\right)$, it is still possible that $\pi_{a_{1}}(X)>$ $\pi_{a_{1}}(b X)+\pi_{a_{2}}((1-b) X)$ for some $0<b<1$. In this case, both reinsurers play a role in the optimal reinsurance design.

Remark 3.3. (i) Let $\mu(b, u)$ be the function defined in (3.2). Note that

$$
\max _{0 \leq b \leq 1} \mu(b, 0)=\mu\left(\frac{a_{1}}{a_{1}+a_{2}}, 0\right)=c-\lambda \frac{a_{1}+a_{2}}{a_{1} a_{2}}\left(M_{Y}\left(\frac{a_{1} a_{2}}{a_{1}+a_{2}}\right)-1\right) .
$$

If

$$
c>\lambda \frac{a_{1}+a_{2}}{a_{1} a_{2}}\left(M_{Y}\left(\frac{a_{1} a_{2}}{a_{1}+a_{2}}\right)-1\right), \quad \text { i.e., } \quad \mu\left(\frac{a_{1}}{a_{1}+a_{2}}, 0\right)>0,
$$

then we can choose $b=a_{1} /\left(a_{1}+a_{2}\right)$ and $u=0$ in $(2.2)$ such that $X_{t}=x+\mu\left(a_{1} /\left(a_{1}+\right.\right.$ $\left.\left.a_{2}\right), 0\right) t$. We can see that there exists arbitrage opportunity in the market. So, we assume that $c \leq \lambda \frac{a_{1}+a_{2}}{a_{1} a_{2}}\left(M_{Y}\left(\frac{a_{1} a_{2}}{a_{1}+a_{2}}\right)-1\right)$. On the other hand, the positive safety loading condition requires that $c>\lambda \mu_{1}$. Therefore, in the rest of this paper, we assume that the following condition holds:

$$
\lambda \mu_{1}<c \leq \lambda \frac{a_{1}+a_{2}}{a_{1} a_{2}}\left(M_{Y}\left(\frac{a_{1} a_{2}}{a_{1}+a_{2}}\right)-1\right) .
$$

(ii) For any $b, u \in[0,1]$, we have

$$
|\mu(b, u)| \leq c+\frac{\lambda}{a_{1}}\left(M_{Y}\left(a_{1}\right)-1\right)+\frac{\lambda}{a_{2}}\left(M_{Y}\left(a_{2}\right)-1\right)+\lambda \mu_{1} \triangleq M .
$$

Then similar to Proposition 3.1 of Cadenillas et al. [3], it is not difficult to derive that

$$
V(x) \leq k(x+|M| / \lambda)
$$

We now present the verification theorem.

Theorem 3.2 (Verification Theorem). Let $v(x) \in C^{1}((0, \infty))$ be a solution to (3.1) at all the points with the possible exception of some point where the second derivative may not exist. Suppose there exists $U>0$ such that $v(x)$ is twice continuously differentiable on $(0, U)$ and $v(x)$ is linear on $[U, \infty)$. Then $V(x) \leq v(x), x \geq 0$. Furthermore, if the $Q V I$ strategy $\alpha^{v}$ associated with $v(x)$ is admissible, then $v(x)$ coincides with the value function $V(x)$ and $\alpha^{v}$ is the optimal strategy, i.e., $V(x)=v(x)=V_{\alpha^{v}}(x), \quad x \geq 0$. 
Proof. Similar to the proof of Theorem 3.4 in Cadenillas et al. [3], it is not difficult to see that equations (3.17) and (3.18) of Cadenillas et al. [3] still hold for our model. So, one can apply Ito's formula (even if the function $v^{\prime \prime}$ might have a discontinuity of the first order at the point $U$ ) to get equations similar to those shown on page 187 of Cadenillas et al. [3], by replacing $\mathcal{L}^{u} v, \mu u_{s}$ and $\sigma u_{s}$ by $\mathcal{L}^{b, u} v-\delta v, \mu\left(b_{s}, u_{s}\right)$ and $\sqrt{\lambda \mu_{2} b_{s}} u_{s}$, respectively. Then, the remaining steps are the same as those in Cadenillas et al. [3].

\section{Solution to the optimization problem}

In order to derive explicit solution to the optimization problem, we consider the following two cases:

(1) $c<\lambda \frac{a_{1}+a_{2}}{a_{1} a_{2}}\left(M_{Y}\left(\frac{a_{1} a_{2}}{a_{1}+a_{2}}\right)-1\right)$;

(2) $c=\lambda \frac{a_{1}+a_{2}}{a_{1} a_{2}}\left(M_{Y}\left(\frac{a_{1} a_{2}}{a_{1}+a_{2}}\right)-1\right)$.

\subsection{Case 1}

\subsubsection{Construction of solution}

In this subsection, we try to construct a solution to (3.1) which satisfies the conditions in Theorem 3.2.

We first assume that there exists a strictly increasing solution $W(x)$ to (3.1) which is continuously differentiable on $(0, \infty)$ and twice continuously differentiable on $\left(0, x_{1}\right)$, where $x_{1}=\inf \{x \geq 0: \mathcal{M} V(x)=V(x)\}$ (all of these will be proved later). Then, (3.1) with $V$ replaced by $W$ for $0 \leq x<x_{1}$ can be rewritten as

$$
\max _{0 \leq b \leq 1,0 \leq u \leq 1}\left\{\frac{1}{2} \lambda \mu_{2} b^{2} u^{2} W^{\prime \prime}(x)+\mu\left(b_{t}, u_{t}\right) W^{\prime}(x)-\delta W(x)\right\}=0 .
$$

Let $b(x)$ and $u(x)$ be the maximizer of the left-hand side of (4.1) over all $b, u \in$ $(-\infty, \infty)$. Differentiating $(4.1)$ with respect to $u$ and $b$ respectively, we get

$$
\begin{aligned}
-\frac{W^{\prime \prime}(x)}{W^{\prime}(x)} & =\frac{M_{Y}^{\prime}\left(a_{2}(1-u(x)) b(x)\right)-\mu_{1}}{\mu_{2} b(x) u(x)}, \\
-\frac{W^{\prime \prime}(x)}{W^{\prime}(x)} & =\frac{M_{Y}^{\prime}\left(a_{1}(1-b(x))\right)-(1-u(x)) M_{Y}^{\prime}\left(a_{2}(1-u(x)) b(x)\right)-u(x) \mu_{1}}{\mu_{2} b(x)[u(x)]^{2}} .
\end{aligned}
$$

Combining (4.2) and (4.3), we have $M_{Y}^{\prime}\left(a_{1}(1-b(x))\right)=M_{Y}^{\prime}\left(a_{2}(1-u(x)) b(x)\right)$. Then,

$$
a_{1}(1-b(x))=a_{2}(1-u(x)) b(x), \quad \text { i.e., } \quad b(x) u(x)=\frac{\left(a_{1}+a_{2}\right) b(x)-a_{1}}{a_{2}} .
$$


Substituting (4.2) and (4.4) into (4.1), we have

$$
g(b(x)) W^{\prime}(x)-\delta W(x)=0 .
$$

where

$$
\begin{aligned}
g(b)=- & \frac{\lambda\left[\left(a_{1}+a_{2}\right) b-a_{1}\right]\left[M_{Y}^{\prime}\left(a_{1}(1-b)\right)-\mu_{1}\right]}{2 a_{2}}+c-\frac{\lambda \mu_{1}\left[\left(a_{1}+a_{2}\right) b-a_{1}\right]}{a_{2}} \\
& -\left(\frac{1}{a_{1}}+\frac{1}{a_{2}}\right) \lambda\left[M_{Y}\left(a_{1}(1-b)\right)-1\right] .
\end{aligned}
$$

Differentiating (4.5) with respect to $x$, we obtain

$$
\left[\frac{d g(b(x))}{d x}-\delta\right] W^{\prime}(x)+g(b) W^{\prime \prime}(x)=0 .
$$

Using (4.2) and (4.4) once again, we have

$$
W^{\prime}(x)\left\{\frac{d g(b(x))}{d x}-\delta-g(b(x)) \frac{a_{2}\left[M_{Y}^{\prime}\left(a_{1}(1-b(x))\right)-\mu_{1}\right]}{\mu_{2}\left[\left(a_{1}+a_{2}\right) b(x)-a_{1}\right]}\right\}=0 .
$$

Since $W^{\prime}(x)>0$, and

$$
\frac{d g(b(x))}{d x}=\frac{\lambda}{2 a_{2}} h(b(x)) b^{\prime}(x),
$$

where

$$
h(b(x))=\left(a_{1}+a_{2}\right)\left[M_{Y}^{\prime}\left(a_{1}(1-b(x))\right)-\mu_{1}\right]+a_{1}\left[\left(a_{1}+a_{2}\right) b(x)-a_{1}\right] M_{Y}^{\prime \prime}\left(a_{1}(1-b(x))\right),
$$

it follows from (4.7) that

$$
b^{\prime}(x)=\frac{2 a_{2}\left\{\delta \mu_{2}\left[\left(a_{1}+a_{2}\right) b(x)-a_{1}\right]+a_{2} g(b(x))\left[M_{Y}^{\prime}\left(a_{1}(1-b(x))\right)-\mu_{1}\right]\right\}}{\lambda \mu_{2}\left[\left(a_{1}+a_{2}\right) b(x)-a_{1}\right] h(b(x))} .
$$

In view of $W(0)=0$ and (4.5), we know that $b(0) \triangleq b_{0}$ is a solution to $g(b)=0$.

Lemma 4.1. The function $g(b)$ is strictly increasing on $\left[a_{1} /\left(a_{1}+a_{2}\right), 1\right]$, and there exists a unique solution $b_{0}$ of $g(b)=0$ on $\left(a_{1} /\left(a_{1}+a_{2}\right), 1\right]$.

Proof. For any $b \in\left[a_{1} /\left(a_{1}+a_{2}\right), 1\right]$, we have

$$
\frac{d g(b)}{d b}=\frac{\lambda}{2 a_{2}}\left\{\left(a_{1}+a_{2}\right)\left[M_{Y}^{\prime}\left(a_{1}(1-b)\right)-\mu_{1}\right]+a_{1}\left[\left(a_{1}+a_{2}\right) b-a_{1}\right] M_{Y}^{\prime \prime}\left(a_{1}(1-b)\right)\right\}>0,
$$

which implies that $g(b)$ is increasing on $\left[a_{1} /\left(a_{1}+a_{2}\right), 1\right]$. Due to Remark 3.1, the result follows from

$$
g\left(\frac{a_{1}}{a_{1}+a_{2}}\right)=\mu\left(\frac{a_{1}}{a_{1}+a_{2}}, 0\right)<0, \quad \text { and } \quad g(1)=c-\lambda \mu_{1}>0
$$


Let

$$
G(b)=\int_{b_{0}}^{b} \frac{\lambda \mu_{2}\left[\left(a_{1}+a_{2}\right) y-a_{1}\right] h(y)}{2 a_{2}\left\{\delta \mu_{2}\left[\left(a_{1}+a_{2}\right) y-a_{1}\right]+a_{2} g(y)\left[M_{Y}^{\prime}\left(a_{1}(1-y)\right)-\mu_{1}\right]\right\}} d y .
$$

Since $g(y)>0$ for all $b_{0}<y \leq 1$, the integrand in the right-hand side of (4.9) is positive on $\left[b_{0}, 1\right]$. It is easy to see that $G(b)$ is increasing on $\left[b_{0}, 1\right]$, which implies that the inverse of $G(b)$ exists on $\left[b_{0}, 1\right]$. Furthermore, it is obvious that $[G(b(x))]^{\prime}=1$, so $b(x)=G^{-1}(x+k)$ for some constant $k$. Since $G\left(b_{0}\right)=0$, we have $k=G\left(b_{0}\right)=0$ which results in

$$
b(x)=G^{-1}(x), \quad 0 \leq x \leq G(1) .
$$

By (4.4), we have

$$
u(x)=\frac{\left(a_{1}+a_{2}\right) b(x)-a_{1}}{a_{2} b(x)} .
$$

Let $b^{*}(x)$ and $u^{*}(x)$ be the maximizer of the left-hand side of (4.1) over all $b, u \in[0,1]$. Since $b(G(1))=u(G(1))=1$, we guess that

$b^{*}(x)=\left\{\begin{array}{cl}G^{-1}(x), & 0 \leq x \leq G(1), \\ 1, & x>G(1),\end{array} \quad\right.$ and $u^{*}(x)=\left\{\begin{array}{cl}\frac{\left(a_{1}+a_{2}\right) G^{-1}(x)-a_{1}}{a_{2} G^{-1}(x)}, & 0 \leq x \leq G(1), \\ 1, & x>G(1) .\end{array}\right.$

For $0 \leq x \leq G(1),(4.2)$ and (4.10) imply that

$$
\left(\ln W^{\prime}(x)\right)^{\prime}=\frac{a_{2}\left[\mu_{1}-M_{Y}^{\prime}\left(a_{1}\left(1-G^{-1}(x)\right)\right)\right]}{\mu_{2}\left[\left(a_{1}+a_{2}\right) G^{-1}(x)-a_{1}\right]},
$$

which leads to

$$
W(x)=q_{1} \int_{0}^{x} \exp \left(\int_{G(1)}^{z} \frac{a_{2}\left[\mu_{1}-M_{Y}^{\prime}\left(a_{1}\left(1-G^{-1}(y)\right)\right)\right]}{\mu_{2}\left[\left(a_{1}+a_{2}\right) G^{-1}(y)-a_{1}\right]} d y\right) d z,
$$

where the constant $q_{1}>0$ will be determined later.

For $G(1)<x \leq x_{1},(4.1)$ becomes

$$
\frac{1}{2} \lambda \mu_{2} W^{\prime \prime}(x)+\left(c-\lambda \mu_{1}\right) W^{\prime}(x)-\delta W(x)=0,
$$

which has the following general solution

$$
W(x)=q_{2} e^{r_{+}(x-G(1))}+q_{3} e^{r_{-}(x-G(1))},
$$

where $q_{2}$ and $q_{3}$ are free constants, and

$$
r_{+}=\frac{-\left(c-\lambda \mu_{1}\right)+\sqrt{\left(c-\lambda \mu_{1}\right)^{2}+2 \lambda \delta \mu_{2}}}{\lambda \mu_{2}}, \quad r_{-}=\frac{-\left(c-\lambda \mu_{1}\right)-\sqrt{\left(c-\lambda \mu_{1}\right)^{2}+2 \lambda \delta \mu_{2}}}{\lambda \mu_{2}} .
$$


For $x>x_{1}$, by the definition of $x_{1}$, we guess that

$$
W(x)=W(\tilde{x})+k(x-\tilde{x})-K,
$$

where $\tilde{x}<x_{1}$ is a constant that needs to be determined below.

We next try to determine the constants $q_{1}, q_{2}, q_{3}, \tilde{x}$ and $x_{1}$. By the continuity of $W^{\prime}$ and $W^{\prime \prime}$ at $G(1)$, we obtain

$$
q_{2} r_{+}+q_{3} r_{-}=q_{1}, \quad q_{2} r_{+}^{2}+q_{3} r_{-}^{2}=0,
$$

which results in $q_{2}=q_{1} b_{1}$ and $q_{3}=q_{1} b_{2}$, where

$$
b_{1}=\frac{r_{-}}{r_{+}\left(r_{-}-r_{+}\right)}>0, \quad b_{2}=\frac{r_{+}}{r_{-}\left(r_{+}-r_{-}\right)}<0 .
$$

Inspired by Bai et al. [2] or Cadenillas et al. [3], we will determine the unknown parameters $q_{1}, \tilde{x}$ and $x_{1}$ in the way that

$$
W^{\prime}(\tilde{x})=W^{\prime}\left(x_{1}\right)=k,
$$

and

$$
\int_{\tilde{x}}^{x_{1}}\left(k-W^{\prime}(y)\right) d y=K .
$$

Define an auxiliary function $U(x)$ as

$$
U(x)= \begin{cases}\exp \left(\int_{G(1)}^{x} \frac{a_{2}\left[\mu_{1}-M_{Y}^{\prime}\left(a_{1}\left(1-G^{-1}(y)\right)\right)\right]}{\mu_{2}\left[\left(a_{1}+a_{2}\right) G^{-1}(y)-a_{1}\right]} d y\right), & 0 \leq x \leq G(1), \\ b_{1} r_{+} e^{r_{+}(x-G(1))}+b_{2} r_{-} e^{r_{-}(x-G(1))}, & x>G(1),\end{cases}
$$

which is equal to $W^{\prime}(x)$ for $0<x \leq x_{1}$. For $x \in[0, G(1))$, it is not difficult to see that

$$
U^{\prime}(x)<0, \quad U^{\prime \prime}(x)>0 .
$$

For $x>G(1)$, we have

$$
\begin{gathered}
U^{\prime}(x)=b_{1} r_{+}^{2} e^{r_{+}(x-G(1))}+b_{2} r_{-}^{2} e^{r_{-}(x-G(1))}=b_{1} r_{+}^{2}\left[e^{r_{+}(x-G(1))}-e^{r_{-}(x-G(1))}\right]>0, \\
U^{\prime \prime}(x)=b_{1} r_{+}^{3} e^{r_{+}(x-G(1))}+b_{2} r_{-}^{3} e^{r_{-}(x-G(1))}>0 .
\end{gathered}
$$

So, the function $U(x)$ is convex on $(0, \infty)$. Since $U^{\prime}(G(1))=0$, the function $U(x)$ attains its minimum at $x=G(1)$ with $U(G(1))=1$. From Figure 1, we have the following conclusions: 


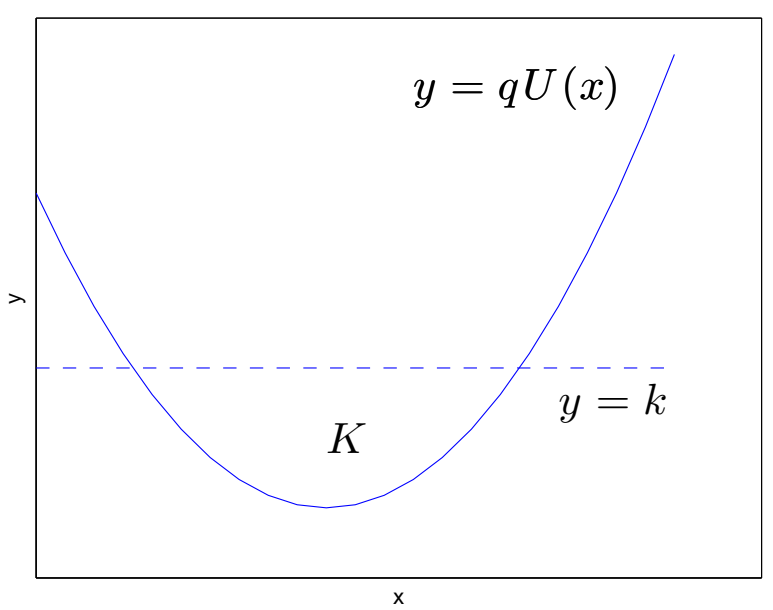

Figure 1: The graph of $q U(x)$. The area between the straight line $y=k$ and the graph of $q U(x)$ is equal to $K$.

(i) For any fixed $q \in(0, k]$, there always exists $\hat{x}_{q} \geq G(1)$ such that $q U\left(\hat{x}_{q}\right)=k$. Furthermore, if $q \downarrow 0$, then $\hat{x}_{q} \uparrow \infty$;

(ii) Let $\bar{q}=k / U(0)<k$. If $q \in[\bar{q}, k], q U(0) \geq k \geq q$, then there exists $\tilde{x}_{q} \in[0, G(1)]$ such that $q U\left(\tilde{x}_{q}\right)=k$. Besides, $\hat{x}_{q}$ is strictly decreasing with respect to $q$; $\tilde{x}_{q}$ is strictly increasing with respect to $q$; and $\hat{x}_{q}=\tilde{x}_{q}=G(1)$ for $q=k$.

Based on (i) and (ii), we consider

$$
I_{1}(q)=\int_{\tilde{x}_{q}}^{\hat{x}_{q}}(k-q U(y)) d y, \quad I_{2}(q)=\int_{0}^{\hat{x}_{q}}(k-q U(y)) d y .
$$

Then, it is not difficult to see that $I_{1}(q)$ is strictly decreasing with respect to $q$ on $[\bar{q}, k]$ and $0=I_{1}(k) \leq I_{1}(q) \leq I_{1}(\bar{q}) \in(0, \infty)$, and that $I_{2}(q)$ is strictly decreasing on $[0, k]$, and

$$
0>\int_{0}^{G(1)} k(1-U(y)) d y=I_{2}(k) \leq I_{2}(q) \leq I_{2}(0)=\infty .
$$

Note that if $I_{1}(\bar{q})>K$, then there exists a unique $q^{*} \in(\bar{q}, k)$ such that $I_{1}\left(q^{*}\right)=K$. Let $x_{1}=\hat{x}_{q^{*}}$ and $\tilde{x}=\tilde{x}_{q^{*}}$. Recalling that for any $x \leq x_{1}, W^{\prime}(x)=q U(x)$, we have

$$
W^{\prime}\left(\tilde{x}_{q^{*}}\right)=W^{\prime}\left(\hat{x}_{q^{*}}\right)=k, W\left(\hat{x}_{q^{*}}\right)=W\left(\tilde{x}_{q^{*}}\right)+k\left(\hat{x}_{q^{*}}-\tilde{x}_{q^{*}}\right)-K ;
$$

and that if $I_{1}(\bar{q}) \leq K$, then there exists a unique $q^{*} \in(0, k)$ such that $I_{2}\left(q^{*}\right)=K$. Let $x_{1}=\hat{x}_{q^{*}}$ and $\tilde{x}=0$. Then, we have

$$
W^{\prime}\left(\hat{x}_{q^{*}}\right)=k, \quad W\left(\hat{x}_{q^{*}}\right)=W(0)+k \hat{x}_{q^{*}}-K .
$$


These together (4.11)-(4.13) yield

$$
W(x)= \begin{cases}q^{*} \int_{0}^{x} \exp \left(\int_{G(1)}^{z} \frac{a_{2}\left[\mu_{1}-M_{Y}^{\prime}\left(a_{1}\left(1-G^{-1}(y)\right)\right)\right]}{\mu_{2}\left[\left(a_{1}+a_{2}\right) G^{-1}(y)-a_{1}\right]} d y\right) d z, & 0 \leq x<G(1), \\ q^{*}\left[b_{1} e^{r+(x-G(1))}+b_{2} e^{r-(x-G(1))}\right], & G(1) \leq x<\hat{x}_{q^{*}}, \\ W\left(\tilde{x}_{q^{*}}\right)+k\left(x-\tilde{x}_{q^{*}}\right)-K, & x \geq \hat{x}_{q^{*}},\end{cases}
$$

where $\tilde{x}_{q^{*}}=0$ if $I_{1}(\bar{q}) \leq K$, and $b_{1}, b_{2}$ are given in (4.14).

Theorem 4.1. The function $W(x)$ of $(4.15)$ is continuously differentiable on $(0, \infty)$ and twice continuously differentiable on $\left(0, \hat{x}_{q^{*}}\right) \cup\left(\hat{x}_{q^{*}}, \infty\right)$. Furthermore, $W(x)$ is a solution to the QVI of (3.1).

Proof. Here, we only prove the case of $I_{1}(\bar{q})>K$. For the case of $I_{1}(\bar{q}) \leq K$, it can be derived using similar arguments. From its construction, it is easy to see that $W(x)$ is continuously differentiable on $(0, \infty)$, and twice continuously differentiable on $\left(0, \hat{x}_{q^{*}}\right) \cup\left(\hat{x}_{q^{*}}, \infty\right)$. To complete the proof, we need to show that $W(x)$ is a solution to the QVI of (3.1).

Similar to the technique of Cadenillas et al. [3], we first prove that $\mathcal{M} W(x)<W(x)$ for $0<x<\hat{x}_{q^{*}}$, and that $\mathcal{M} W(x)=W(x)$ for $x>\hat{x}_{q^{*}}$.

Since $U^{\prime}(x)<0$ for $0<x<G(1)$, we see that $W^{\prime}(x)=q^{*} U(x)$ is a strictly decreasing function on $[0, G(1)]$. Let $f(\eta)=W(x-\eta)+k \eta-K, 0<\eta \leq x$. Note that $W^{\prime}\left(\tilde{x}_{q^{*}}\right)=k$. Hence, for any $x \leq \tilde{x}_{q^{*}}$, we have $f^{\prime}(\eta)=-W^{\prime}(x-\eta)+k<0$, which in turn yields

$$
\mathcal{M} W(x)=\sup _{0<\eta \leq x} f(\eta)=f(0+)=W(x)-K<W(x) .
$$

For $\tilde{x}_{q^{*}} \leq x<\hat{x}_{q^{*}}, f^{\prime}\left(x-\tilde{x}_{q^{*}}\right)=-W^{\prime}\left(\tilde{x}_{q^{*}}\right)+k=0$, then we obtain

$$
\mathcal{M} W(x)=f\left(x-\tilde{x}_{q^{*}}\right)=W\left(\tilde{x}_{q^{*}}\right)+k\left(x-\tilde{x}_{q^{*}}\right)-K=W\left(\hat{x}_{q^{*}}\right)-k\left(\hat{x}_{q^{*}}-x\right)<W(x),
$$

where the last inequality follows from $W^{\prime}(x)<k$ for any $x \in\left(\tilde{x}_{q^{*}}, \hat{x}_{q^{*}}\right)$.

We now show that $\mathcal{M} W(x)=W(x)$ for $x>\hat{x}_{q^{*}}$. If $\eta \in\left(0, x-\hat{x}_{q^{*}}\right]$, then

$W(x-\eta)+k \eta-K=W\left(\tilde{x}_{q^{*}}\right)+k\left(x-\eta-\tilde{x}_{q^{*}}\right)-K+k \eta-K=W(x)-K<W(x)$.

If $\eta \in\left(x-\hat{x}_{q^{*}}, x\right]$, then

$$
\begin{aligned}
W(x-\eta)+k \eta-K & =W\left(\hat{x}_{q^{*}}-\left[\eta-\left(x-\hat{x}_{q^{*}}\right)\right]\right)+k\left[\eta-\left(x-\hat{x}_{q^{*}}\right)\right]-K+k\left(x-\hat{x}_{q^{*}}\right) \\
& \leq W\left(\hat{x}_{q^{*}}\right)+k\left(x-\hat{x}_{q^{*}}\right)=W(x),
\end{aligned}
$$

where the equality holds if and only if $\eta=x-\tilde{x}_{q^{*}}$. So, we have $\mathcal{M} W(x)=W(x)$ for $x>\hat{x}_{q^{*}}$. 
We next prove that

$$
\begin{cases}\max _{0 \leq b \leq 1,0 \leq u \leq 1} \mathcal{L}^{b, u} W(x)-\delta W(x)=0, & 0<x<\hat{x}_{q^{*}} \\ \max _{0 \leq b \leq 1,0 \leq u \leq 1} \mathcal{L}^{b, u} W(x)-\delta W(x)<0, & x>\hat{x}_{q^{*}}\end{cases}
$$

For $0 \leq x<G(1)$, we only need to prove that $W(x)$ satisfies (4.5) with $b(x)=$ $G^{-1}(x)$. From its construction, we know that $W(x)$ satisfies (4.6). This implies that $W(x)$ should satisfy (4.5) with a constant (not necessarily equal to 0 ) on the right-hand side. Since $g\left(b_{0}\right)=0, W(0)=0$ and

$$
\begin{aligned}
W^{\prime}(0) & =q^{*} \exp \left(\int_{0}^{G(1)} \frac{a_{2}\left[M_{Y}^{\prime}\left(a_{1}\left(1-G^{-1}(y)\right)\right)-\mu_{1}\right]}{\mu_{2}\left[\left(a_{1}+a_{2}\right) G^{-1}(y)-a_{1}\right]} d y\right) \\
& \leq q^{*} \exp \left(\frac{a_{2}\left[M_{Y}^{\prime}\left(a_{1}\right)-\mu_{1}\right] G(1)}{\mu_{2}\left[\left(a_{1}+a_{2}\right) b_{0}-a_{1}\right]}\right)<\infty,
\end{aligned}
$$

the right-hand side of (4.5) tends to 0 when $x \rightarrow 0$. It follows that $W(x)$ satisfies (4.5) for all $0 \leq x<G(1)$.

For $G(1) \leq x \leq \hat{x}_{q^{*}}$,

$$
W^{\prime}(x)=q^{*} U(x) \geq q^{*}>0, W^{\prime \prime}(x)=q^{*} U^{\prime}(x)>0 .
$$

Then, for any fixed $b \in[0,1]$, we have

$$
\frac{\partial \mathcal{L}^{b, u} W(x)}{\partial u}=\lambda \mu_{2} b^{2} u W^{\prime \prime}(x)+\lambda b\left[M_{Y}^{\prime}\left(a_{2}(1-u) b\right)-\mu_{1}\right] W^{\prime}(x)>0, \quad \forall u \in[0,1] .
$$

Therefore,

$\mathcal{L}^{b, u} W(x) \leq \mathcal{L}^{b, 1} W(x)=\frac{1}{2} \lambda \mu_{2} b^{2} W^{\prime \prime}(x)+\left[c-\frac{\lambda}{a_{1}}\left(M_{Y}\left(a_{1}(1-b)\right)-1\right)-\lambda \mu_{1} b\right] W^{\prime}(x)$.

On the other hand,

$$
\frac{\partial \mathcal{L}^{b, 1} W(x)}{\partial b}=\lambda \mu_{2} b W^{\prime \prime}(x)+\lambda\left[M_{Y}^{\prime}\left(a_{1}(1-b)\right)-\mu_{1}\right] W^{\prime}(x)>0, \forall b \in[0,1] .
$$

As a result, we obtain

$$
\max _{0 \leq b \leq 1,0 \leq u \leq 1} \mathcal{L}^{b, u} W(x)=\mathcal{L}^{1,1} W(x)=\frac{1}{2} \lambda \mu_{2} W^{\prime \prime}(x)+\left(c-\lambda \mu_{1}\right) W^{\prime}(x) .
$$

Finally, it follows from the construction of $W(x)$ that

$$
\max _{0 \leq b \leq 1,0 \leq u \leq 1} \mathcal{L}^{b, u} W(x)-\delta W(x)=\frac{1}{2} \lambda \mu_{2} W^{\prime \prime}(x)+\left(c-\lambda \mu_{1}\right) W^{\prime}(x)-\delta W(x)=0 .
$$


For $x>\hat{x}_{q^{*}}$, since $W(x)=W\left(\tilde{x}_{q^{*}}\right)+k\left(x-\tilde{x}_{q^{*}}\right)-K$, we have

$$
\begin{aligned}
& \max _{0 \leq b \leq 1,0 \leq u \leq 1} \mathcal{L}^{b, u} W(x)-\delta W(x)=k \max _{0 \leq b \leq 1,0 \leq u \leq 1} \mu(b, u)-\delta W(x) \\
= & k \mu(1,1)-\delta W(x)=k\left(c-\lambda \mu_{1}\right)-\delta W(x)<k\left(c-\lambda \mu_{1}\right)-\delta W\left(\hat{x}_{q^{*}}\right) \\
< & \frac{1}{2} \lambda \mu_{2} W^{\prime \prime}\left(\hat{x}_{q^{*}}-\right)+k\left(c-\lambda \mu_{1}\right)-\delta W\left(\hat{x}_{q^{*}}\right) \\
= & \mathcal{L}^{1,1} W\left(\hat{x}_{q^{*}}-\right)-\delta W\left(\hat{x}_{q^{*}}\right)=0 .
\end{aligned}
$$

Hence, (4.16) holds.

4.1.2. The value function and the optimal policy

Let

$$
b_{t}^{*}=\left\{\begin{array}{cl}
G^{-1}\left(X_{t}^{*}\right), & 0 \leq X_{t}^{*} \leq G(1), \\
1, & X_{t}^{*}>G(1),
\end{array} \quad u_{t}^{*}=\left\{\begin{array}{cl}
\frac{\left(a_{1}+a_{2}\right) G^{-1}\left(X_{*}^{*}\right)-a_{1}}{a_{2} G^{-1}\left(X_{t}^{*}\right)}, & 0 \leq X_{t}^{*} \leq G(1), \\
1, & X_{t}^{*}>G(1),
\end{array}\right.\right.
$$

and $\left\{\tau_{n}^{*}, \xi_{n}^{*}, n \geq 1\right\}$ are defined as follows:

(i) If $I_{1}(\bar{q})>K$, then we define

$$
\tau_{1}^{*}=\inf \left\{t>0: X_{t}^{*}=\hat{x}_{q^{*}}\right\}, \quad \xi_{1}^{*}=\hat{x}_{q^{*}}-\tilde{x}_{q^{*}},
$$

when the initial surplus $0<x<\hat{x}_{q^{*}}$,

$$
\tau_{1}^{*}=0, \quad \xi_{1}^{*}=x-\tilde{x}_{q^{*}},
$$

when the initial surplus $x \geq \hat{x}_{q^{*}}$, and

$$
\tau_{n}^{*}=\inf \left\{t>\tau_{n-1}^{*}: X_{t}^{*}=\hat{x}_{q^{*}}\right\}, \xi_{n}^{*}=\hat{x}_{q^{*}}-\tilde{x}_{q^{*}},
$$

for every $n \geq 2$, where $X_{t}^{*}$ is given by

$$
X_{t}^{*}=x+\int_{0}^{t} \mu\left(b_{t}^{*}, u_{t}^{*}\right) d t+\int_{0}^{t} \sqrt{\lambda \mu_{2}} b_{t}^{*} u_{t}^{*} d W_{t}-\left(\hat{x}_{q^{*}}-\tilde{x}_{q^{*}}\right) \sum_{n=1}^{\infty} I_{\left(\tau_{n}^{*}<t\right)},
$$

when the initial surplus $0<x<\hat{x}_{q^{*}}$, and

$$
X_{t}^{*}=x+\int_{0}^{t} \mu\left(b_{t}^{*}, u_{t}^{*}\right) d t+\int_{0}^{t} \sqrt{\lambda \mu_{2}} b_{t}^{*} u_{t}^{*} d W_{t}-\left(x-\tilde{x}_{q^{*}}\right) I_{\left(\tau_{1}^{*}<t\right)}-\left(\hat{x}_{q^{*}}-\tilde{x}_{q^{*}}\right) \sum_{n=2}^{\infty} I_{\left(\tau_{n}^{*}<t\right)},
$$

when the initial surplus $x \geq \hat{x}_{q^{*}}$; 
(ii) If $I_{1}(\bar{q}) \leq K$, then we define

$$
\tau_{1}^{*}=\inf \left\{t>0: X_{t}^{*}=\hat{x}_{q^{*}}\right\}, \quad \xi_{1}^{*}=\hat{x}_{q^{*}},
$$

when the initial surplus $0<x<\hat{x}_{q^{*}}$,

$$
\tau_{1}^{*}=0, \quad \xi_{1}^{*}=x
$$

when the initial surplus $x \geq \hat{x}_{q^{*}}$, and

$$
\tau_{n}^{*}=\infty, \quad \xi_{n}^{*}=0,
$$

for every $n \geq 2$, where $X_{t}^{*}$ is given by

$$
X_{t}^{*}=x+\int_{0}^{t} \mu\left(b_{t}^{*}, u_{t}^{*}\right) d t+\int_{0}^{t} \sqrt{\lambda \mu_{2}} b_{t}^{*} u_{t}^{*} d W_{t}, \quad t \leq \tau_{1}^{*},
$$

when the initial surplus $0<x<\hat{x}_{q^{*}}$.

Theorem 4.2. The value function $V(x)$ is given by (4.15) and the strategy $\alpha^{*}=$ $\left(b_{t}^{*} ; u_{t}^{*} ; \tau_{1}^{*}, \tau_{2}^{*}, \cdots ; \xi_{1}^{*}, \xi_{2}^{*}, \cdots\right)$ is the corresponding optimal policy.

Proof. It follows from Definition 3.1 and the arguments in the proof of Theorem 4.1 that $\alpha^{*}=\left(b_{t}^{*} ; u_{t}^{*} ; \tau_{1}^{*}, \tau_{2}^{*}, \cdots ; \xi_{1}^{*}, \xi_{2}^{*}, \cdots\right)$ defined above is the QVI strategy associated with $W(x)$ which is given by (4.15). Besides, it is easy to see that $\alpha^{*}$ is admissible. Hence, the optimal result is an immediate consequence of Theorem 3.2.

\subsection{Case 2}

In this subsection, we consider Case 2 with

$$
c=\lambda \frac{a_{1}+a_{2}}{a_{1} a_{2}}\left(M_{Y}\left(\frac{a_{1} a_{2}}{a_{1}+a_{2}}\right)-1\right) .
$$

To show that $W(x)$ of $(4.15)$ is the value function, and that $\alpha^{*}$ in Theorem 4.2 is the optimal policy, one can apply arguments similar to those used in the previous subsection. However, from Lemma 4.1, we know that $b_{0}=a_{1} /\left(a_{1}+a_{2}\right)$ in Case 2 . Consequently, the integrand on the right-hand side of (4.9) and (4.11) might have a singularity. Therefore, we need to show that the integrals in the right-hand side of (4.9) and (4.11) make sense in this case.

\section{Proposition 4.3.}

$$
\lim _{x \rightarrow 0} b^{\prime}(x)=\frac{2 a_{2} \delta \mu_{2}+\lambda a_{2}\left[M_{Y}^{\prime}\left(\frac{a_{1} a_{2}}{a_{1}+a_{2}}\right)-\mu_{1}\right]^{2}}{\lambda \mu_{2}\left(a_{1}+a_{2}\right)\left[M_{Y}^{\prime}\left(\frac{a_{1} a_{2}}{a_{1}+a_{2}}\right)-\mu_{1}\right]} .
$$


Proof. Note that $b(x) \rightarrow a_{1} /\left(a_{1}+a_{2}\right)$ and $g(b(x)) \rightarrow g\left(a_{1} /\left(a_{1}+a_{2}\right)\right)=0$ as $x \rightarrow 0$. Applying l'Hospital's rule to the right-hand side of (4.8), we get

$$
\begin{aligned}
\lim _{x \rightarrow 0} b^{\prime}(x) & =\lim _{b \rightarrow \frac{a_{1}}{a_{1}+a_{2}}} \frac{2 a_{2}\left\{\delta \mu_{2}\left[\left(a_{1}+a_{2}\right) b-a_{1}\right]+a_{2} g(b)\left[M_{Y}^{\prime}\left(a_{1}(1-b)\right)-\mu_{1}\right]\right\}}{\lambda \mu_{2}\left[\left(a_{1}+a_{2}\right) b-a_{1}\right] h(b)} \\
& =\frac{2 a_{2}}{\lambda \mu_{2}} \frac{\delta \mu_{2}\left(a_{1}+a_{2}\right)+a_{2} g^{\prime}\left(\frac{a_{1}}{a_{1}+a_{2}}\right)\left[M_{Y}^{\prime}\left(\frac{a_{1} a_{2}}{a_{1}+a_{2}}\right)-\mu_{1}\right]}{\left(a_{1}+a_{2}\right)^{2}\left[M_{Y}^{\prime}\left(\frac{a_{1} a_{2}}{a_{1}+a_{2}}\right)-\mu_{1}\right]} \\
& =\frac{2 a_{2} \delta \mu_{2}+\lambda a_{2}\left[M_{Y}^{\prime}\left(\frac{a_{1} a_{2}}{a_{1}+a_{2}}\right)-\mu_{1}\right]^{2}}{\lambda \mu_{2}\left(a_{1}+a_{2}\right)\left[M_{Y}^{\prime}\left(\frac{a_{1} a_{2}}{a_{1}+a_{2}}\right)-\mu_{1}\right]} .
\end{aligned}
$$

Proposition 4.4. Let $G(b)$ be given in (4.9). Then, $G(1)<\infty$.

Proof. Since

$$
G(1)=\int_{\frac{a_{1}}{a_{1}+a_{2}}}^{1} \frac{\lambda \mu_{2}\left[\left(a_{1}+a_{2}\right) y-a_{1}\right] h(y)}{2 a_{2}\left\{\delta \mu_{2}\left[\left(a_{1}+a_{2}\right) y-a_{1}\right]+a_{2} g(y)\left[M_{Y}^{\prime}\left(a_{1}(1-y)\right)-\mu_{1}\right]\right\}} d y
$$

and the integrand in the above expression tends to

$$
\frac{\lambda \mu_{2}\left(a_{1}+a_{2}\right)\left[M_{Y}^{\prime}\left(\frac{a_{1} a_{2}}{a_{1}+a_{2}}\right)-\mu_{1}\right]}{2 a_{2} \delta \mu_{2}+\lambda a_{2}\left[M_{Y}^{\prime}\left(\frac{a_{1} a_{2}}{a_{1}+a_{2}}\right)-\mu_{1}\right]^{2}}, \quad y \rightarrow \frac{a_{1}}{a_{1}+a_{2}} .
$$

Hence, the results follows from Proposition 4.3.

Proposition 4.5. Let $W(x)$ be given in (4.11). Then,

$$
W^{\prime}(x)=q_{1} \exp \left(\int_{G(1)}^{x} \frac{a_{2}\left[\mu_{1}-M_{Y}^{\prime}\left(a_{1}(1-b(y))\right)\right]}{\mu_{2}\left[\left(a_{1}+a_{2}\right) b(y)-a_{1}\right]} d y\right) \sim x^{-n}, \quad x \rightarrow 0,
$$

where

$$
n=\frac{\lambda\left[M_{Y}^{\prime}\left(\frac{a_{1} a_{2}}{a_{1}+a_{2}}\right)-\mu_{1}\right]^{2}}{2 \delta \mu_{2}+\lambda\left[M_{Y}^{\prime}\left(\frac{a_{1} a_{2}}{a_{1}+a_{2}}\right)-\mu_{1}\right]^{2}}<1,
$$

and the notation $f(x) \sim g(x)$ means that $f(x) / g(x) \rightarrow c_{1}$ for some constant $c_{1}>0$ as $x \rightarrow 0$.

Proof. It follows from (4.17) that

$$
b(x)-b(0)=\frac{2 a_{2} \delta \mu_{2}+\lambda a_{2}\left[M_{Y}^{\prime}\left(\frac{a_{1} a_{2}}{a_{1}+a_{2}}\right)-\mu_{1}\right]^{2}}{\lambda \mu_{2}\left(a_{1}+a_{2}\right)\left[M_{Y}^{\prime}\left(\frac{a_{1} a_{2}}{a_{1}+a_{2}}\right)-\mu_{1}\right]} x+o(x), x \rightarrow 0 .
$$

As a result, we obtain

$$
\begin{aligned}
W^{\prime}(x) & \sim \exp \left(\int_{G(1)}^{x} \frac{a_{2}\left[\mu_{1}-M_{Y}^{\prime}\left(a_{1}(1-b(y))\right)\right]}{\mu_{2}\left[\left(a_{1}+a_{2}\right) b(y)-a_{1}\right]} d y\right) \\
& \sim \exp \left(-\int_{G(1)}^{x} \frac{a_{2}\left[M_{Y}^{\prime}\left(\frac{a_{1} a_{2}}{a_{1}+a_{2}}\right)-\mu_{1}\right]^{2} \lambda \mu_{2}}{\mu_{2}\left(2 a_{2} \delta \mu_{2}+\lambda a_{2}\left[M_{Y}^{\prime}\left(\frac{a_{1} a_{2}}{a_{1}+a_{2}}\right)-\mu_{1}\right]^{2}\right.} \frac{1}{y} d y\right) \\
& \sim x^{-\frac{\lambda\left[M_{Y}^{\prime}\left(\frac{a_{1} a_{2}}{a_{1}+a_{2}}\right)-\mu_{1}\right]^{2}}{2 \delta \mu_{2}+\lambda\left[M_{Y}^{\prime}\left(\frac{a_{1} a_{2}}{a_{1}+a_{2}}\right)-\mu_{1}\right]^{2}}}, \quad x \rightarrow 0 .
\end{aligned}
$$


According to Proposition 4.5, we have the integrability at 0 of the integrand on the right-hand side of (4.11). Besides, from the proof of Theorem 4.1, we should verify that the right-hand side of (4.5) tends to 0 when $x \rightarrow 0$. Due to (4.18) and (4.19), we have

$$
W^{\prime}(x) \sim\left(b(x)-\frac{a_{1}}{a_{1}+a_{2}}\right)^{-n}, \quad x \rightarrow 0 .
$$

Therefore, it is sufficient to show that

$$
\lim _{b \rightarrow \frac{a_{1}}{a_{1}+a_{2}}} g(b)\left(b-\frac{a_{1}}{a_{1}+a_{2}}\right)^{-n}=0 .
$$

Applying l'Hospital's rule, we get

$$
\begin{aligned}
& \lim _{b \rightarrow \frac{a_{1}}{a_{1}+a_{2}}}\left[c-\left(\frac{1}{a_{1}}+\frac{1}{a_{2}}\right) \lambda\left[M_{Y}\left(a_{1}(1-b)\right)-1\right]\left(b-\frac{a_{1}}{a_{1}+a_{2}}\right)^{-n}\right. \\
= & \lim _{b \rightarrow \frac{a_{1}}{a_{1}+a_{2}}} \frac{\lambda\left(a_{1}+a_{2}\right) M_{Y}^{\prime}\left(a_{1}(1-b)\right)}{a_{2} n\left(b-\frac{a_{1}}{a_{1}+a_{2}}\right)^{n-1}}=0,
\end{aligned}
$$

which implies that the right-hand side of (4.5) tends to 0 as $x \rightarrow 0$.

\section{Some comments}

The problem studied in Chen et al. [4] can be extended to the case of two reinsurers, which is also the case without transaction costs of this paper. Take the unbounded dividend rates for example. Following the arguments in Chen et al. [4], we know that the value function $V(x)$ satisfies (4.1) for $0 \leq x<x_{1}$ and $V^{\prime}(x)=1$ for $x \geq x_{1}$, where $x_{1}=\inf \left\{x \geq 0: V^{\prime}(x) \leq 1\right\}=G(1)$. Since $V^{\prime}\left(x_{1}\right)=1$, it is easy to see that $q_{1}=1$ in (4.11) and $V\left(x_{1}\right)=\frac{c-\lambda \mu_{1}}{\delta}$ by (4.5). Therefore, the value function $V(x)$ is given by

$$
V(x)= \begin{cases}\int_{0}^{x} \exp \left(\int_{G(1)}^{z} \frac{a_{2}\left[\mu_{1}-M_{Y}^{\prime}\left(a_{1}\left(1-G^{-1}(y)\right)\right)\right]}{\mu_{2}\left[\left(a_{1}+a_{2}\right) G^{-1}(y)-a_{1}\right]} d y\right) d z, & 0 \leq x \leq G(1), \\ \frac{c-\lambda \mu_{1}}{\delta}+x-G(1), & x>G(1)\end{cases}
$$

and the optimal reinsurance strategy is given by (4.10) .

\section{Numerical example}

The influence of $k$ and $K$ on the critical levels $\hat{x}_{q}$ and $\tilde{x}_{q}$ are clear from Figure 1. Since the effects of $a_{1}$ and $a_{2}$ (risk aversion parameters of the reinsurers) on the critical levels $\hat{x}_{q}$ and $\tilde{x}_{q}$ are rather complicated, we give a numerical example to illustrate the effects of $a_{1}$ and $a_{2}$ on the optimal reinsurance strategy in this section. In the example, 
we assume that the claim sizes are exponentially distributed with parameter 1 , and set $\lambda=1, c=3 / 2$, and $\delta=0.05$. By fixing $a_{1}=1$ and taking $a_{2}=0.6,0.8,1,1.5,2$, the optimal proportions on $[0, G(1)]$ for the insurer and two reinsurers are exhibited in Figures 2-4, and the values of $G(1)$ are given in Table 1 .

From Figures 2-4, we see that the effect of $a_{2}$ wears off as $a_{2}$ increases. Figures 2 and 4 show that, when $a_{2}$ changes, the impact on the optimal proportions of the insurer and the second reinsurer is significant for small initial surplus, and becomes weaker for large initial surplus. Finally, we observe from Figure 3 that for the first reinsurer, the impact of $a_{2}$ on $1-b^{*}(x)$ increases to a certain level as the initial surplus increases, and remains at that level for large initial surplus (the lines in Figure 3 are almost parallel when the initial surplus is more than 1.5).

\begin{tabular}{|c|c|c|c|c|c|}
\hline$a_{2}$ & 0.6 & 0.8 & 1 & 1.5 & 2 \\
\hline$G(1)$ & 3.8242 & 4.3072 & 4.5250 & 4.8028 & 4.8402 \\
\hline
\end{tabular}

Table 1: The values of $G(1)$ for $a_{1}=1$

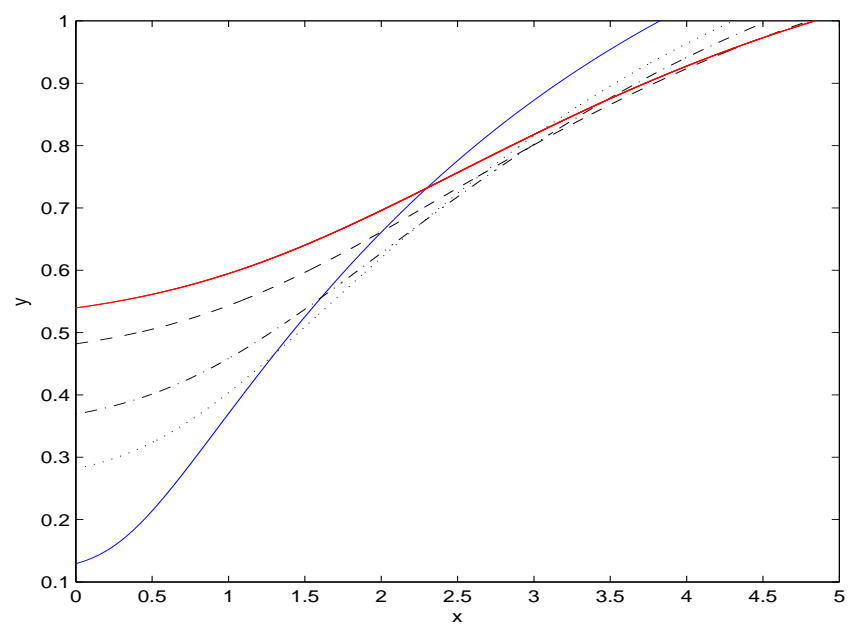

Figure 2: The optimal retention level function $y=u^{*}(x) b^{*}(x)$ of the insurer for $a_{2}=0.6,0.8,1,1.5,2$ from bottom to top (at the beginning of the function)

\section{Acknowledgements}

The research of Mi Chen was supported by National Natural Science Foundation of China (Nos. 11426063, 11371020 and 11171164) and Natural Science Foundation of Fujian Province (Nos. 2015J05003 and JA14077). The research of Kam C. Yuen was 


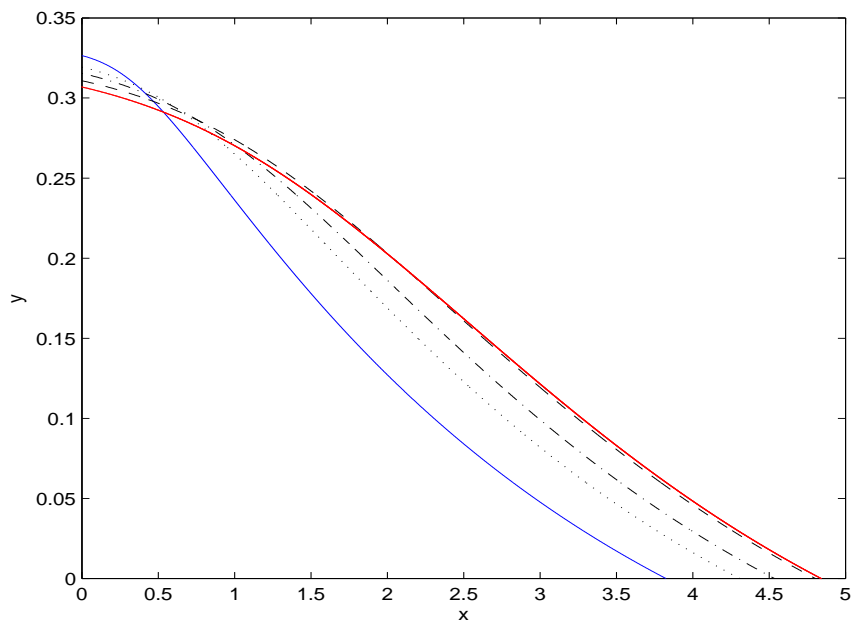

Figure 3: The first reinsurer's optimal reinsurance proportion function $y=1-b^{*}(x)$ for $a_{2}=$ $0.6,0.8,1,1.5,2$ from bottom to top (at the beginning of the function)

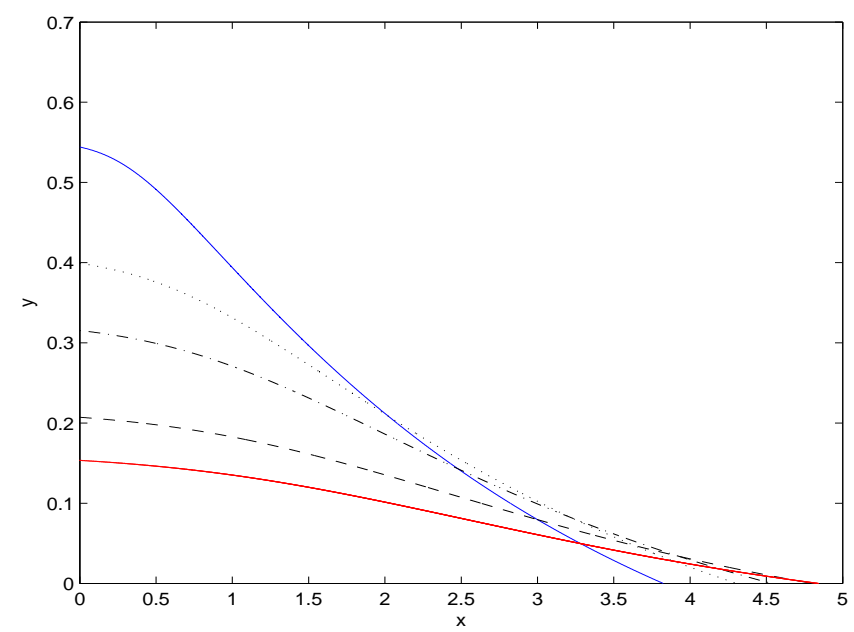

Figure 4: The second reinsurer's optimal reinsurance proportion function $y=\left(1-u^{*}(x)\right) b^{*}(x)$ for $a_{2}=0.6,0.8,1,1.5,2$ from bottom to top (at the beginning of the function) 
supported by a grant from the Research Grants Council of the Hong Kong Special Administrative Region, China (Project No. HKU 7057/13P), and the CAE 2013 research grant from the Society of Actuaries - any opinions, finding, and conclusions or recom-

mendations expressed in this material are those of the authors and do not necessarily reflect the views of the SOA.

\section{References}

[1] Asimit, A. V., Badescu, A. M., Verdonck, T., 2013. Optimal risk transfer under quantile-based risk measurers. Insurance: Mathematics and Economics 53 (1), 252-265.

[2] Bai, L., Guo, J., Zhang, H., 2010. Optimal excess-of-loss reinsurance and dividend payments with both transaction costs and taxes. Quantitative Finance 10 (10), $1163-1172$.

[3] Cadenillas, A., Choulli, T., Taksar, M., Zhang, L., 2006. Classical and impulse stochastic control for the optimization of the dividend and risk policies of an insurance firm. Mathematical Finance 16 (1), 181-202.

[4] Chen, M., Peng, X., Guo, J., 2013. Optimal dividend problem with a nonlinear regular-singular stochastic control. Insurance: Mathematics and Economics 52 (3), $448-456$.

[5] Chi, Y., Meng, H., 2014. Optimal reinsurance arrangements in the presence of two reinsurers. Scandinavian Actuarial Journal 5, 424-438.

[6] Choulli, T., Taksar, M., Zhou, X., 2001. Excess-of-loss reinsurance for a company with debt liability and constraints on risk reduction. Quantitative Finance 1 (6), 573-596.

[7] de Finetti, B., 1957. Su un'impostazione alternativa della teoria collettiva del rischio. Transactions of the XVth International Congress of Actuaries 2 (1), 433443.

[8] Fleming, W. H., Soner, H. M., 2006. Controlled Markov processes and viscosity solutions. Vol. 25. Springer Verlag. 
[9] Guan, H., Liang, Z., 2014. Viscosity solution and impulse control of the diffusion model with reinsurance and fixed transaction costs. Insurance: Mathematics and Economics 54, 109-122.

[10] Guo, X., Liu, J., Zhou, X. Y., 2004. A constrained non-linear regular-singular stochastic control problem, with applications. Stochastic processes and their applications 109 (2), 167-187.

[11] He, L., Liang, Z., 2008. Optimal financing and dividend control of the insurance company with proportional reinsurance policy. Insurance: Mathematics and Economics $42(3), 976-983$.

[12] He, L., Liang, Z., 2009. Optimal financing and dividend control of the insurance company with fixed and proportional transaction costs. Insurance: Mathematics and Economics 44 (1), 88-94.

[13] Kaluszka, M., 2001. Optimal reinsurance under mean-variance premium principles. Insurance: Mathematics and Economics 28 (1), 61-67.

[14] Kaluszka, M., 2005. Optimal reinsurance under convex principles of premium calculation. Insurance: Mathematics and Economics 36 (3), 375-398.

[15] Løkka, A., Zervos, M., 2008. Optimal dividend and issuance of equity policies in the presence of proportional costs. Insurance: Mathematics and Economics 42 (3), 954-961.

[16] Meng, H., Siu, T. K., 2011. Optimal mixed impulse-equity insurance control problem with reinsurance. SIAM Journal on Control and Optimization 49 (1), 254-279.

[17] Meng, H., Siu, T. K., 2011. On optimal reinsurance, dividend and reinvestment strategies. Economic Modelling 28 (1), 211-218.

[18] Moore, K. S., Young, V. R., 2003. Pricing equity-linked pure endowments via the principle of equivalent utility. Insurance: Mathematics and Economics 33 (3), $497-516$.

[19] Musiela, M., Zariphopoulou, T., 2004. An example of indifference prices under exponential preferences. Finance and Stochastics 8 (2), 229-239. 
[20] Peng, X., Chen, M., Guo, J., 2012. Optimal dividend and equity issuance problem with proportional and fixed transaction costs. Insurance: Mathematics and Economics 51 (3), 576-585.

[21] Scheer, N., Schmidli, H., 2011. Optimal dividend strategies in a Cramer-Lundberg model with capital injections and administration costs. European Actuarial Journal 1 (1), 57-92.

[22] Schmidli, H., 2002. On minimizing the ruin probability by investment and reinsurance. The Annals of Applied Probability 12 (3), 890-907.

[23] Yao, D., Yang, H., Wang, R., 2014. Optimal risk and dividend control problem with fixed costs and salvage value: variance premium principle. Economic Modelling $37,53-64$.

[24] Young, V. R., 1999. Optimal insurance under wang's premium principle. Insurance: Mathematics and Economics 25 (2), 109-122.

[25] Young, V. R., 2003. Equity-indexed life insurance: pricing and reserving using the principle of equivalent utility. North American Actuarial Journal 7 (1), 68-86.

[26] Young, V. R., Zariphopoulou, T., 2002. Pricing dynamic insurance risks using the principle of equivalent utility. Scandinavian Actuarial Journal 2002 (4), 246-279.

[27] Zhou, M., Yuen, K. C., 2012. Optimal reinsurance and dividend for a diffusion model with capital injection: variance premium principle. Economic Modelling 29 (2), 198-207.

MI CHEN, Fujian Normal University

School of Mathematics and Computer Science, Fujian Normal University, Fuzhou 350108, China.

KAM C. YUEN, The University of Hong Kong

Department of Statistics and Actuarial Science, The University of Hong Kong, Pokfulam Road, Hong Kong.

E-mail: kcyuen@hku.hk 